\title{
Communication
}

\section{The transformation from (111) to (100): A routine to large-scale epitaxial diamond}

\author{
Yang Wang ${ }^{1, *}$, Weihua Wang ${ }^{1}$, Shilin Yang ${ }^{1}$ and Jiaqi Zhu ${ }^{1,2}$ \\ 1 Center for Composite Materials and Structures, Harbin Institute of Technology, Harbin150080; P.R. China; \\ weihuawang2011@163.com (W.W.); 18846089791@163.com (S.Y.); zhujq@hit.edu.cn (J.Z.) \\ 2 Key Laboratory of Micro-systems and Micro-structures Manufacturing, Ministry of Education, Harbin \\ 150080; P.R. China \\ * Correspondence: ravimassa@163.com; Tel.: +86-1524-462-0495
}

\begin{abstract}
Diamond is a material with excellent performances which attracts the attention from researchers for decades. $\mathrm{Pt}$ (111), owing to its catalytic activity on diamond synthesis, is regarded to be a candidate for diamond hetero-epitaxity, which can enhance nucleation density. Molten surface at diamond growth temperature can also improve mobility and aggregation capability of primitive nuclei. Generally, (100)-oriented is welcomed for the achivement of high quality and large size diamond, since the formation of defects and twins are prevented. First-principle calculations and experimental researches were carried out for the study of transformation of orientation. The transformation from $\{111\}$ to $\{100\}$-oriented diamond has been observed on $\mathrm{Pt}$ (111) substrate, which can be promoted by the increase of carbon source concentration and substrate temperature. The process is energetic favorable, which may provides a way towards large-scale (100) diamond films.
\end{abstract}

Keywords: diamond film; Pt (111) substrate; orientation transformation; formation energy

\section{Introduction}

Consisted of $\mathrm{C}$ sp3 bonds $\mathrm{s}^{[1]}$, diamond possesses plenty of outstanding performances, like high hardness ${ }^{[2]}$, thermal conductivity ${ }^{[3]}$ and structural stability ${ }^{[4]}$, thus can be applied in a variety of fields, such as tools ${ }^{[5]}$, heat $\sin ^{[6]}$, sensors ${ }^{[7,8]}$ and optoelectronic devices ${ }^{[9,10]}$ for high-tech industry. Taking structural integrity into consideration, epitaxial films and even single crystal diamonds (SCD) are in great need ${ }^{[11]}$, owing to the lack of grain boundaries and less impurities ${ }^{[12]}$.

There are two approaches towards SCD, namely High Pressure High Temperature (HPHT) and Chemical Vapor Deposition (CVD) methods ${ }^{[13]}$. The harsh synthesis conditions of HPHT is a barrier preventing the further expansion of crystal size, while CVD provides a solution to this problem ${ }^{[14]}$. To bypass the size limitation of diamond seeds, hetero-epitaxity is regarded an option, then, choosing a proper foreign substrate will be the first step.

The catalytic performance of substrate materials has a positive effect on the primitive nuclei formation and nucleation density of diamond. Despite a large number of alternatives, such as $\mathrm{Si}{ }^{[15,16]}$, $\mathrm{SiC}[17]$, or $\operatorname{Ir}\left[{ }^{18-20]}, \mathrm{Pt}[21,22]\right.$, with a strong catalytic effect on hydrocarbons, is often used as the metal catalyst for the synthesis of HPHT diamond, which can greatly increase the nucleation density of diamond, so that the nucleation stage can proceed directly without bias to reduce substrate damage. It can also prevent the formation of graphite in the initial stage of CVD, providing an excellent bonding strength with the sediment. In addition, during the nucleation stage of CVD diamond, due to the melting state of $\mathrm{Pt}$ surface, the position and orientation of small diamond particles can easily change and adjust, which benefits the orientation consistency[23].

However, although $\operatorname{Pt}(111)$ has been commercialized as a standard substrate for the growth of ferroelectric materials ${ }^{[24,25]}$, difficulties are still exist in the preparation of $\operatorname{Pt}(100)$ substrate. If the transformation from diamond $\{111\}$ to $\{100\}$ can be achieved during growth process, large-size and high quality epitaxial diamond films are expected to obtain. 


\section{Calculation and Experimental Details}

\subsection{Models and First-principle Calculations Details}

The model of diamond unit cell whose corner atoms were cut off to expose $\{111\}$ crystal faces was created. Then the corner atoms were added subsequently until a complete structure with the outer surfaces of $\{100\}$ was achieved, and the formation energies were recorded during the process.

First-principle calculations were performed by CASTEP based on density functional theory (DFT). Generalized gradient approximation (GGA) method was used to cover the geometry optimization, and Perdew, Burke, Ernzerhof (PBE) functional was employed for exchange-correlation energy. The interaction between ions and valence electrons was described by OTFG ultrasoft pseudopotential. To calculate interfacial structures, the cut-off energy was set to $400 \mathrm{eV}$, and the Monkhorst-Pack k-point sampling was chosen as $5 \times 5 \times 5$ grid. In addition, two adjacent slabs are separated by placing in a cube box with a side length of $10 \AA$ to avoid interaction between slabs and its period images. The convergence tolerances of energy, maximum force, stress, and displacement were set to $1 \times 10^{-5} \mathrm{eV} /$ atom, $0.03 \mathrm{eV} / \AA ̊ \Omega, 0.05 \mathrm{GPa}$ and $0.001 \AA$, respectively.

\subsection{Experimental Details}

The $10 \mathrm{~mm} \times 10 \mathrm{~mm} \times 0.5 \mathrm{~mm} \mathrm{Pt}(111)(200 \mathrm{~nm}) / \mathrm{Ti}(50 \mathrm{~nm}) / \mathrm{SiO} 2(500 \mathrm{~nm}) / \mathrm{Si}(100)$ wafers purchased from Hefei Kejing Materials Technology CO.,LTD, Hefei, China was employed as the substrates of diamond growth. The diamond films were deposited in a $2.45 \mathrm{GHz}, 6 \mathrm{~kW}$ Ardis 300 coaxial cavity resonator type microwave plasma chemical vapour deposition (MPCVD) research device. Since methane concentration and substrate temperature are the two most important factors affecting the facet index of diamond crystals surface, these two parameters were changed and detailed conditions for each sample are shown in Table 1.

Table 1. Detailed preparation conditions for diamond film samples.

\begin{tabular}{cccccc}
\hline $\begin{array}{c}\text { Sample } \\
\text { Number }\end{array}$ & $\begin{array}{c}\text { Methane } \\
\text { Concentration } \\
\text { /\% }\end{array}$ & $\begin{array}{c}\text { Pressure } \\
\text { /mbar }\end{array}$ & $\begin{array}{c}\text { Substrate } \\
\text { Temperature } \\
/{ }^{\circ} \mathbf{C}\end{array}$ & $\begin{array}{c}\text { Microwave } \\
\text { Power } / \mathbf{W}\end{array}$ & $\begin{array}{c}\text { Deposite } \\
\text { Time } / \mathbf{h}\end{array}$ \\
\hline 1 & 2 & & 850 & & \\
2 & 3 & 112 & 850 & & 30 \\
3 & 4 & & 850 & 2500 & \\
4 & 4 & & 800 & & \\
5 & 4 & 900 & & \\
\hline
\end{tabular}

The structure tests were carried out by an Empyrean X-ray diffractometer with a thin film analysis accessory made by the Dutch company Panalytical so that the grazing incidence $X$-ray diffraction (GIXRD) pattern can be measured. The Raman spectrometer HORIBA Jobin-Yvon LabRAM HR Evolution by HORIBA Scientific was used to analyze the quantity of deposit. HELIOS NanoLab 600i scanning electron microscope by FEI, America was employed to investigate surface topography.

\section{Results and Discussion}

\subsection{Formation energy in transformation from diamond (111) to (100)}

To get further insight into the outer surfaces of CVD diamond crystals, growth rate ratio of (100) and (111) facets $\alpha$ is usually defined as:

$$
\alpha=\sqrt{3} \frac{V_{(100)}}{V_{(111)}}
$$

$V_{(100)}$ and $V_{(111)}$ stand for the growth rates of (100) and (111) facets respectively, which are controlled by different precursors, namely $\left[\mathrm{CH}_{3}\right]$ and $\left[\mathrm{C}_{2} \mathrm{H}_{2}\right]$. When $\left[\mathrm{C}_{2} \mathrm{H}_{2}\right] /\left[\mathrm{CH}_{3}\right]$ is higher than a certain value, making $\alpha \leq 1 / 3$, (111) facets grow at a high speed, resulting in the exposure of (100) facets completely. 
The ratio $\left[\mathrm{C}_{2} \mathrm{H}_{2}\right] /\left[\mathrm{CH}_{3}\right]$ is related to substrate temperature and methane concentration, so changes in these two parameters can accelerate the transformation of preferred orientation.

The atomic structure evolution as well as step-by-step formation energy during transformation from diamond (111) to (100) was also investigated, in which process, $C$ atoms are gradually attached on the surface of diamond $\{111\}$ crystal planes and eventually achieve a structure covered with $\{100\}$ sufaces. The formation energy can be calculated as Equation (2):

$$
\Delta E_{\text {step-by-step }}=E_{n}-\left(E_{n-1}+E_{\text {carbon }}\right),
$$

$\Delta E_{\text {step-by-step }}$ is the energy difference caused by the attachment and bonding of new carbon atoms, $E_{n}$ and $E_{n-1}$ are the energies of the newly formed and previous configurations after or before adsorption, while Ecarbon stands for the energy of a single carbon atom adsorbed during each step. From Figure 1 , the energy is decreasing at each step, indicating that the gradual growth of the film is a spontaneous exothermic process.

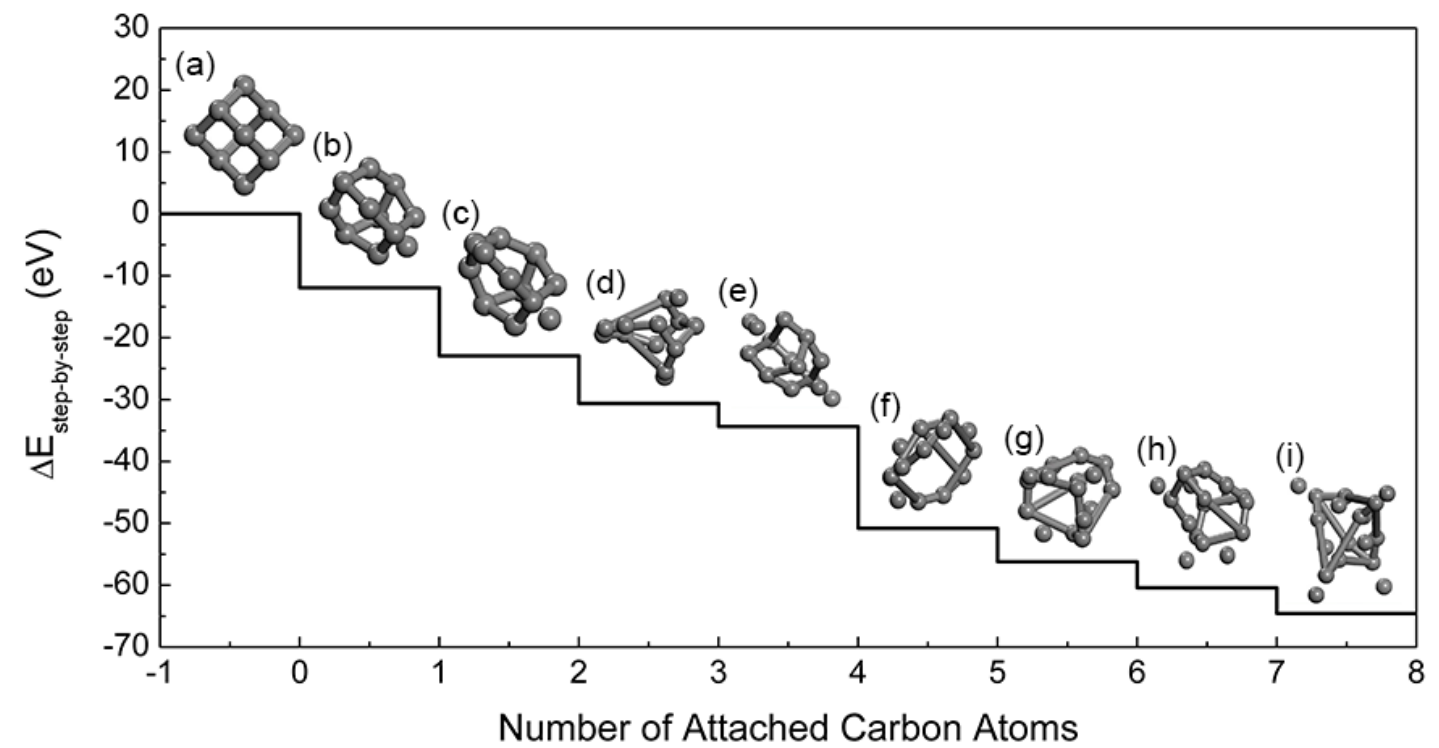

Figure 1. (Colour online) Energy profile of the transformation from diamond $\{111\}$ to $\{100\}$ step-bystep. The clean surface is set as the reference state with $\Delta E_{\text {step-by-step }}=0 \mathrm{eV}$. The insets (a)-(i) are stable configurations of each step, grey balls are the previous carbon cluster, while the orange ones are the newly-attached carbon atoms.

\subsection{Nucleation of diamond on Pt(111)}

Nucleation is the first step to diamond hetero-epitaxity. A high nucleation density is an essential if large-scale and high quality epitaxial film is required. In order to investigate the nucleation process of diamond on $\mathrm{Pt}$ (111) substrate, the growth conditions (substrate temperature and methane concentration) of Sample 3\# were carried out while the sample was taken out and tested to understand the nucleation process after 2, 11 and 21 hours, respectively. The morphologies are shown in Figure 2.

Figure 2a illustrates the formation of nuclei, although not so large in quantity, the aggregation and self-assembly of nuclei can be found, which is of extreme significance for the formation of secondary nuclei in the subsequent stage. Although the self-assembly of particles is very common in liquid, it is not easy to observe on solid surface due to limited movement. However, the possibility of this process is greatly increased because of the fact that Pt surface can be kept in a molten state at diamond growth temperature. 
As the deposition time goes on, the grain density and size raise obviously, and eventually develop into a continuous film. If defects has been preset before deposition (such as the crack in Figure $2 b$ ), the nuclei will tend to accumulate here, and this is an effective way to enhance nucleation density.
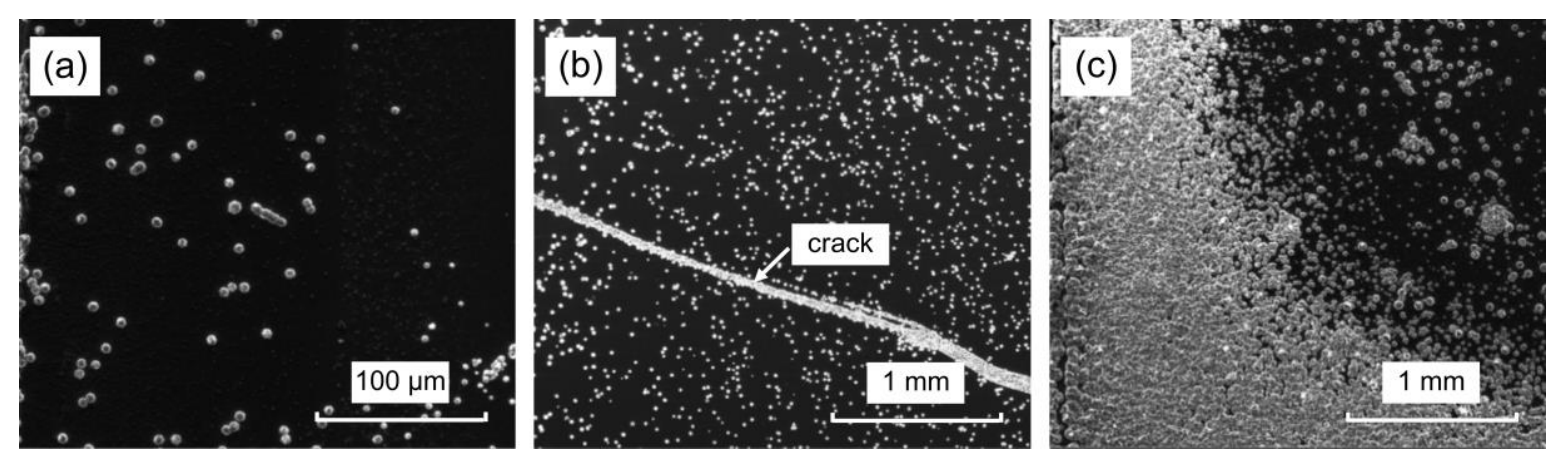

Figure 2 (Colour: no need) SEM images of the process of formation of diamond nuclei on $\mathrm{Pt}$ (111) with $\left(\mathrm{CH}_{4} / \mathrm{H}_{2}\right) \%=4 \%$ and growth temperature $850^{\circ} \mathrm{C}$, the deposition time was (a) $2 \mathrm{~h}$; (b) $11 \mathrm{~h}$; (c) $21 \mathrm{~h}$, respectively. Note the difference in the scale of (a).

Compared with the conventional diamond growth substrates (such as $\mathrm{Si}$, etc.), even without the imposition of bias voltage, a relatively high nucleation density on $\mathrm{Pt}$ substrate can be acquired. Different from the nucleation domain on Ir substrate, the nucleation of diamond on Pt substrate is more uniform. Therefore, there is no amorphous region between domains during expanding and joining. It would also be easier for the accumulation and adjustment of crystals, which is conducive to a regular structure and orientation consistency.

\subsection{Effects of methane concentration and substrate temperature}

Carbon source concentration is an important factor affecting the quality and orientation of CVD diamond. The ratio of gas flow rate of carbon source gas $\mathrm{CH}_{4}$ to that of $\mathrm{H}_{2}$ (that is, $\left(\mathrm{CH}_{4} / \mathrm{H}_{2}\right) \%$ ) is usually taken as the standard. Diamond films deposited at the same temperature but under three different carbon source concentrations (2\%-1\#,3\%-2\# and 4\%-3\#) were characterized and analyzed to explore the influence on diamond nucleation, growth and orientation.

Raman spectrum can reflect the purity of diamond film, that is, the content of diamond phase in the sample, as shown in Figure 3b. The characteristic peaks at $1332.5 \mathrm{~cm}^{-1}$ represent the attendance of diamond in each sample. XRD can accurately distinguish all kinds of crystal orientation and intensity, therefore identifying texture and preferential orientation. Figure 3a shows the XRD spectra of diamond films. When $\left(\mathrm{CH}_{4} / \mathrm{H}_{2}\right) \%=2 \%$, (111) is the main diffraction peak, with another (220) peak but (100) signal is absent. With the rise of $\mathrm{CH}_{4}$ concentration, especially when $\left(\mathrm{CH}_{4} / \mathrm{H}_{2}\right) \%=4 \%$, (100) facets begin to take dominant position, and the diffraction peak intensity of diamond (111) was decreased sharply, indicating that the highly (100) oriented diamond film is obtained, which can also be verified by SEM image.
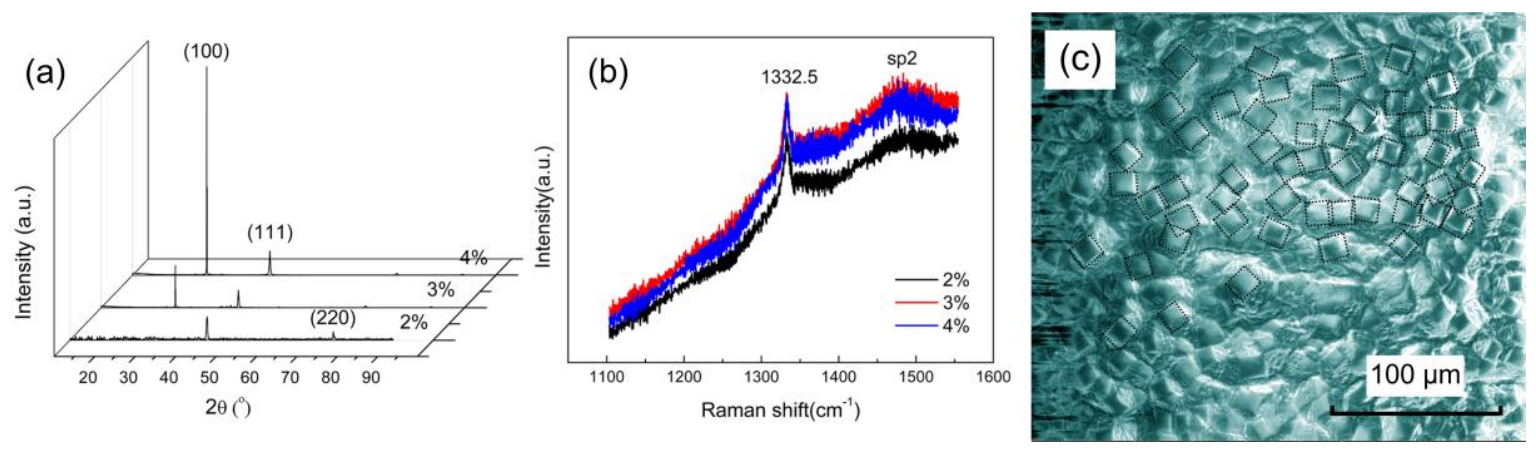

Figure 3 (Colour online) Effects of carbon source concentration $\left(\mathrm{CH}_{4} / \mathrm{H}_{2}\right) \%$ on diamond films deposited on Pt (111) substrate. (a) XRD spectra of samples $1 \#-3 \#$ with different $\left(\mathrm{CH}_{4} / \mathrm{H}_{2}\right) \%=2 \%, 3 \%$ 
and 4\% respectively; (b) Raman spectra; (c) SEM image of sample 3\# ( $\left(\mathrm{CH}_{4} / \mathrm{H}_{2}\right) \%=4 \%$, deposition temperature $850{ }^{\circ} \mathrm{C}$ ), on which the squares marked by dotted lines represent some of the $(100)$ oriented grains.

In Figure 3c, quite a number of cubic grains can be identified, which represent the (100) oriented crystals. Therefore, with lower concentration of carbon source, the diamond crystals grown on $\mathrm{Pt}$ (111) substrate are mainly (111) oriented. As carbon source concentration increases, these (111) oriented grains gradually grow and transform into (100) orientation.

Substrate temperature is another contributing factor for the quality of CVD diamond films. At different temperatures, competitive growth mechanisms will work at various crystal facets, so different orientation will be achieved. From the Raman spectra in Figure $4 \mathrm{a}$, the characteristic peaks at $\sim 1332 \mathrm{~cm}^{-1}$ representing diamond phase in the samples grown at $800{ }^{\circ} \mathrm{C}(4 \#), 850{ }^{\circ} \mathrm{C}(3 \#)$ and 900 ${ }^{\circ} \mathrm{C}(5 \#)$ with the same $\left(\mathrm{CH}_{4} / \mathrm{H}_{2}\right) \%=4 \%$. With the increase of growth temperature to 850 or $900{ }^{\circ} \mathrm{C}$, the intensity of peaks corresponding to the amorphous carbon decreased significantly compared with that at $800{ }^{\circ} \mathrm{C}$.

Comparing the SEM images showing the surface morphology of samples at different growth temperatures, in the case of $800{ }^{\circ} \mathrm{C}$, as shown in Figure $4 \mathrm{~b}$, there are triangular and rhombic grains on the surface. The triangular ones are that of $\{111\}$-oriented facets, while the rhombic grains are the morphology of twin formation. However, at the temperature of $850{ }^{\circ} \mathrm{C}$ (Figure 3c) and $900{ }^{\circ} \mathrm{C}$ (Figure $4 \mathrm{c}$ ), almost all the outer surface of diamond grains are $\{100\}$-oriented, which demonstrate cubic shapes. When the temperature increases to $900{ }^{\circ} \mathrm{C}$, a lot of grains with similar orientations have been connected together, and there is a tendency of the formation of continuous film. In addition, some trapezoidal grains can be seen, which is the intermediate state from $\{111\}$ to $\{100\}$ orientation, indicating that the final $\{100\}$-oriented grains are transformed from those of $\{111\}$-oriented.
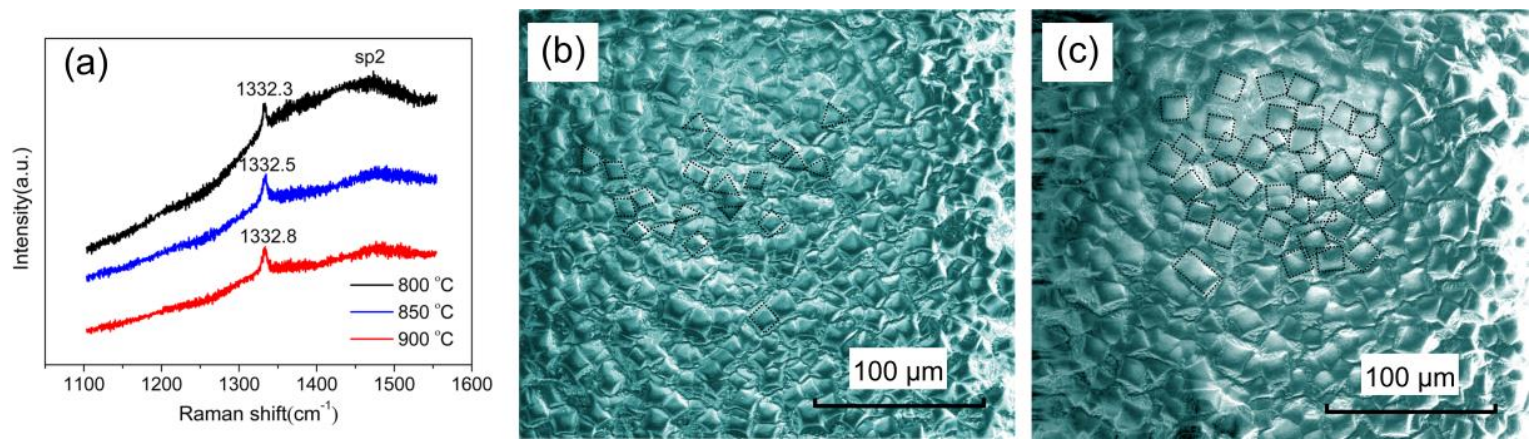

Figure 4 (Colour online) Effects of substrate temperature on the morphology of diamond films on $\mathrm{Pt}$ (111). (a) Raman spectra of samples 3\#-5\# with different substrate temperature 800,850 and $900{ }^{\circ} \mathrm{C}$ respectively, in which $(\mathrm{CH} 4 / \mathrm{H} 2) \%=4 \%$; SEM images of diamond film deposited at (b) $800{ }^{\circ} \mathrm{C}$, triangular and rhombic marked by dotted lines represent some of the (111) oriented grains or twincrystals; and (c) $900^{\circ} \mathrm{C}$, the square marked by dotted lines represent some of the (100) oriented grains.

\section{Conclusions}

In this paper, the transformation of diamond (111) to (100) orientation has been studied by both first-principle calculations and experimental investigations, and the conclusions are as follows:

(1) The transformation from $\{111\}$ to $\{100\}$ for diamond grains outer surface orientation is spontaneous and of energetic favorableness;

(2) Diamond can nucleate on Pt (111) substrate with high density and uniformity, while the accumulation of nuclei can be observed;

(3) The increase of $\mathrm{CH} 4$ concentration and growth temperature can promote the rapid growth and pinching-out of $\{111\}$ facets, resulting in the exposure of $\{100\}$ facets, thus achieving highlyoriented diamond (100) films.

Due to the commercialization of high quality $\mathrm{Pt}$ (111) substrates whose diameter can reach 5 inches, the conversion may become a promising approach towards large-scale diamond (100) films. 
Author Contributions: Writing - original draft preparation: Y.W.; measurement and data analysis: Y.W., W.W., and S.Y.; methodology: Y.W.; supervision: J.Z. All authors have read and agreed to the published version of the manuscript.

Funding: This research was funded by National Natural Science Foundation of China, grant numbers 51702066, 51911530123, National Science Fund for Distinguished Young Scholars, grant number 51625201.

Acknowledgments: Y.W. especially wants to say sorry to her 3-year-old son, Novak Zheng, for not being with him for nearly half a year, hoping this paper can become a gift for his fourth birthday.

Conflicts of Interest: The authors declare no conflict of interest.

\section{References}

1. Chen, N.; Zhang, G.; Li, R.; Xu, G.; Wang, F.; Ma, H.; Jia, X. Defect and stress reduction in high-pressure and high-temperature synthetic diamonds using gradient cooling technology. Cryst. Growth Des. 2020, 20, 3358-3364.

2. Fang, X.; Yang, Z.; Tan, S.; Duan, L. Feasibility study of selective laser melting for metal matrix diamond tools. Crystals 2020, 10, 596.

3. Muchnikov, A.B.; Radishev, D.B.; Vikharev, A.L.; Gorbachev, A.M.; Mitenkin, A.V.; Drozdov, M.N.; Drozdov, Y.N.; Yunin, P.A. Characterization of interfaces in mosaic CVD diamond crystal. J.Cryst. Growth 2016, 442, 62-67.

4. Ichikawa, K.; Kodama, H.; Suzuki, K.; Sawabe, A. Dislocation in heteroepitaxial diamond visualized by hydrogen plasma etching. Thin Solid Films 2016, 600, 142-145.

5. Yu, Y.; Wu, L.; Zhi, J. Diamond nanowires: fabrication, structure, properties, and applications. Angew Chem. Int. Ed. Engl. 2014, 53, 14326-14351.

6. Zhang, R.; Zhao, W.S.; Yin, W.Y.; Zhao, Z.G.; Zhou, H.J. Impacts of diamond heat spreader on the thermomechanical characteristics of high-power AlGaN/GaN HEMTs. Diam. Relat. Mater. 2015, 52, 25-31.

7. Smirnov, W.; Kriele, A.; Yang, N.; Nebel, C.E. Aligned diamond nano-wires: Fabrication and characterisation for advanced applications in bio- and electrochemistry. Diam. Relat. Mater. 2010, 19, 186189.

8. Gurbuz, Y.; Esame, O.; Tekin, I.; Kang, W.P.; Davidson, J.L. Diamond semiconductor technology for RF device applications. Solid-State Electron. 2005, 49, 1055-1070.

9. Kasu, M. Diamond epitaxy: Basics and applications. Prog. Cryst. Growth Ch. 2016, 62, 317-328.

10. Wang, J.J.; He, Z.Z.; Yu, C.; Song, X.B.; Wang, H.X.; Lin, F.; Feng, Z.H. Comparison of field-effect transistors on polycrystalline and single-crystal diamonds. Diam. Relat. Mater. 2016, 70, 114-117.

11. Kampfer, S.; Cho, N.; Combs, S.E.; Wilkens, J.J. Dosimetric characterization of a single crystal diamond detector in X-ray beams for preclinical research. Z. Med. Phys. 2018, 28, 303-309.

12. Yamada, H.; Chayahara, A.; Mokuno, Y.; Kato, Y.; Shikata, S. Effects of crystallographic orientation on the homoepitaxial overgrowth on tiled single crystal diamond clones. Diam. Relat. Mater. 2015, 57, 17-21.

13. Bolshakov, A.P.; Ralchenko, V.G.; Yurov, V.Y.; Popovich, A.F.; Antonova, I.A.; Khomich, A.A.; Ashkinazi, E.E.; Ryzhkov, S.G.; Vlasov, A.V.; Khomich, A.V. High-rate growth of single crystal diamond in microwave plasma in $\mathrm{CH} 4 / \mathrm{H} 2$ and $\mathrm{CH} 4 / \mathrm{H} 2 /$ Ar gas mixtures in presence of intensive soot formation. Diam. Relat. Mater. 2016, 62, 49-57.

14. Boussadi, A.; Tallaire, A.; Kasu, M.; Barjon, J.; Achard, J. Reduction of dislocation densities in single crystal CVD diamond by confinement in the lateral sector. Diam. Relat. Mater. 2018, 83, 162-169.

15. Robertson, J.; Gerber, J.; Sattel, S.; Weiler, M.; Jung, K.; Ehrhardt, H. Mechanism of bias-enhanced nucleation of diamond on Si. Appl. Phys. Lett. 1995, 66, 3287-3289.

16. Jiang, X.; Jia, C.L. Diamond epitaxy on (001) silicon: An interface investigation. Appl. Phys. Lett. 1995, 67, 1197-1199.

17. Suto, T.; Yaita, J.; Iwasaki, T.; Hatano, M. Highly oriented diamond (111) films synthesized by pulse biasenhanced nucleation and epitaxial grain selection on a 3C-SiC-Si (111) substrate. Appl. Phys. Lett. 2017, 110, 062102.

18. Fischer, M.; Brescia, R.; Gsell, S.; Schreck, M.; Brugger, T.; Greber, T.; Osterwalder, J.; Stritzker, B. Growth of twin-free heteroepitaxial diamond on Ir/YSZ/Si(111). J. Appl. Phys. 2008, 104, 1072-1082.

19. Gallheber, B.-C.; Fischer, M.; Mayr, M.; Straub, J.; Schreck, M. Growth, stress, and defects of heteroepitaxial diamond on Ir/YSZ/Si(111). J. Appl. Phys. 2018, 123, 225302. 
20. Chavanne, A.; Arnault, J.C.; Barjon, J.; Arabski, J. Bias-enhanced nucleation of diamond on iridium: A comprehensive study of the first stages by sequential surface analysis. Surf. Sci. 2011, 605, 564-569.

21. Hayashi, Y.; Matsushita, Y.; Soga, T.; Umeno, M.; Jimbo, T. The formation of a (111) texture of the diamond film on $\mathrm{Pt} / \mathrm{TiO} 2 / \mathrm{SiOx} / \mathrm{Si}$ substrate by microwave plasma chemical vapor deposition. Diam. Relat. Mater. 2002, 11, 499-503.

22. Honda, K.; Yoshimura, M.; Rao, T.N.; Tryk, D.A.; Fujishima, A.; Yasui, K.; Sakamoto, Y.; Nishio, K.; Masuda, H. Electrochemical properties of Pt-modified nano-honeycomb diamond electrodes. J. Electroanalytical Chem. 2001, 514, 35-50.

23. Tachibana, T.; Yokota, Y.; Miyata, K.; Onishi, T.; Kobashi, K.; Tarutani, M.; Takai, Y.; Shimizu, R.; Shintani, Y. Diamond films heteroepitaxially grown on platinum (111). Phys. Rev. B 1997, 56, 88423-15981.

24. William, R.V.; Marikani, A.; Madhavan, D. Dielectric behavior and magnetical response for porous BFO thin films with various thicknesses over Pt/Ti/SiO2/Si substrate. Ceram. Int. 2016, 42, 6807-6816.

25. Lu, Y.W.; Shieh, J.; Tsai, F.Y. Induction of ferroelectricity in nanoscale $\mathrm{ZrO} 2 / \mathrm{HfO} 2$ bilayer thin films on $\mathrm{Pt} / \mathrm{Ti} / \mathrm{SiO} 2 / \mathrm{Si}$ substrates. Acta Mater. 2016, 115, 68-75. 